\title{
Differences Between the Iterated Prisoner's Dilemma and the Chicken Game under Noisy Conditions
}

\author{
Bengt Carlsson \\ Dept. of Software Engineering and Computer Science \\ Blekinge Institute of Technology \\ S-372 25 Ronneby, Sweden \\ +46457385813 \\ bengt.carlsson@bth.se
}

\author{
K. Ingemar Jönsson \\ Department of Theoretical Ecology \\ Lund University, Ecology Building \\ S-223 62 Lund, Sweden. \\ +46462223771 \\ ingemar.jonsson@teorekol.lu.se
}

\begin{abstract}
The prisoner's dilemma has evolved into a standard game for analyzing the success of cooperative strategies in repeated games. With the aim of investigating the behavior of strategies in some alternative games we analyzed the outcome of iterated games for both the prisoner's dilemma and the chicken game. In the chicken game, mutual defection is punished more strongly than in the prisoner's dilemma, and yields the lowest fitness. We also ran our analyses under different levels of noise. The results reveal a striking difference in the outcome between the games. Iterated chicken game needed more generations to find a winning strategy. It also favored nice, forgiving strategies able to forgive a defection from an opponent. In particular the well-known strategy tit-for-tat has a poor successrate under noisy conditions. The chicken game conditions may be relatively common in other sciences, and therefore we suggest that this game should receive more interest as a cooperative game from researchers within computer science.
\end{abstract}

Keywords: Game theory, prisoner's dilemma, chicken game, noise, tit-for-tat

\section{INTRODUCTION}

Iterated games have become a popular tool for analyzing social behavior and cooperation based on reciprocity in multi agent systems ([3], [4], [5), [8)). By allowing games to be played several times and against several other strategies a "shadow of the future", i.e. a non-zero probability for the agents to meet again in the future, is created for the current game. This increases the opportunity for cooperative behavior to evolve (e.g., [5)).

Most iterative analyses on cooperation have focused on the payoff environment defined as the prisoner's dilemma (PD) ([4], [8), [12] , [17]). In terms of payoffs, a PD is defined when $T>R>P>S$, and $2 \mathrm{R}>\mathrm{T}+\mathrm{S}$ according to Figure 1a. The second condition means that the value of the payoff, when shared in cooperation, must be greater than it is when shared by a cooperator and a defector. Because it pays more to defect, no matter how the opponent

Permission to make digital or hard copies of all or part of this work for personal or classroom use is granted without fee provided that copies are not made or distributed for profit or commercial advantage, and that copies bear this notice and the full citation on the first page. To copy otherwise, to republish, to post on servers or to redistribute to lists, requires prior specific permission and/or a fee. chooses to act, an agent is bound to defect, if the agents are not deriving advantage from repeating the game. If $2 \mathrm{R}<\mathrm{T}+\mathrm{S}$ is allowed there will be no upper limit for the value of the temptation. However, there is no definite reason for excluding this possibility. Carlsson and Johansson [9] argued that Rapoport and Chammah [20] introduced this constraint for practical more than theoretical reasons. PD belongs to a class of games where each player has a dominating strategy of playing defect in the single play PD.

\begin{tabular}{|l|l|l|l|l|l|l|}
\cline { 2 - 3 } \cline { 5 - 5 } \multicolumn{1}{l|}{} & Cooperate & Defect & \multicolumn{1}{c|}{ b. } & Cooperare & Defect \\
\hline $\begin{array}{l}\text { Coop- } \\
\text { erate }\end{array}$ & $\mathrm{R}$ & $\mathrm{S}$ & \begin{tabular}{|l|l|l|} 
Coop- \\
erate
\end{tabular} & 1 & $(\mathrm{~S}-\mathrm{P}) /(\mathrm{R}-\mathrm{P})$ \\
\hline Defect & $\mathrm{T}$ & $\mathrm{P}$ & Defect & $(\mathrm{T}-\mathrm{P}) /(\mathrm{R}-\mathrm{P})$ & 0 \\
\hline
\end{tabular}

Figure 1. Pay-off matrices for $2 * 2$ games where $R=$ reward, $S$ $=$ sucker, $T=$ temptation and $P=$ punishment. In $b$ the four variables $R, S, T$ and $P$ are reduced to two variables $S^{\prime}=(S-P) /$ $(\mathrm{R}-\mathrm{P})$ and $\mathrm{T}^{\prime}=(\mathrm{T}-\mathrm{P}) /(\mathrm{R}-\mathrm{P})$.

Chicken game (CG) is a similar but much less studied game than $\mathrm{PD}$, and is defined when $\mathrm{T}>\mathrm{R}>\mathrm{S}>\mathrm{P}$. Mutual defection is thus punished more in the CG than in the PD. In the single-play form, the CG has no dominant strategy (although it has two Nash equilibria in pure strategies, and one mixed equilibrium), and thus no expected outcome as in the PD [13]. Together with the generous chicken game (GCG), often called the battle of sexes [14], CG belongs to a class of games where neither player has a dominating strategy. For a GCG, playing defect increases the payoff for both of them, unless the other agent also plays $\operatorname{defect}(\mathrm{T}>\mathrm{S}>\mathrm{R}>\mathrm{P})$.

In Figure $1 \mathrm{~b}, \mathrm{R}$ and $\mathrm{P}$ are assumed to be fixed to 1 and 0 respectively. This can be obtained through a two steps reduction where all variables are first subtracted by $\mathrm{P}$ and then divided by R-P. This makes it possible to describe the games with only two parameters $\mathrm{S}^{\prime}$ and $\mathrm{T}^{\prime}$. In fact we can capture all possible $2 \times 2$ games in a twodimensional plane.

In Figure 2 the parameter space for PD, CG and GCG defined by $S^{\prime}$ and $T^{\prime}$, is shown. T' $=1$ marks a dividing line between conflict and cooperation. $\mathrm{S}^{\prime}=0$ marks the line between $\mathrm{CG}$ and $\mathrm{PD} . \mathrm{T}^{\prime}<1$ means that playing cooperate $(\mathrm{R})$ is favored over playing defect (T) when the other agent cooperates. This prevents an agent from being "selfish" in a surrounding of cooperation. Conflicting games are expected when T' $>1$ because of better outcome playing temptation (T). 


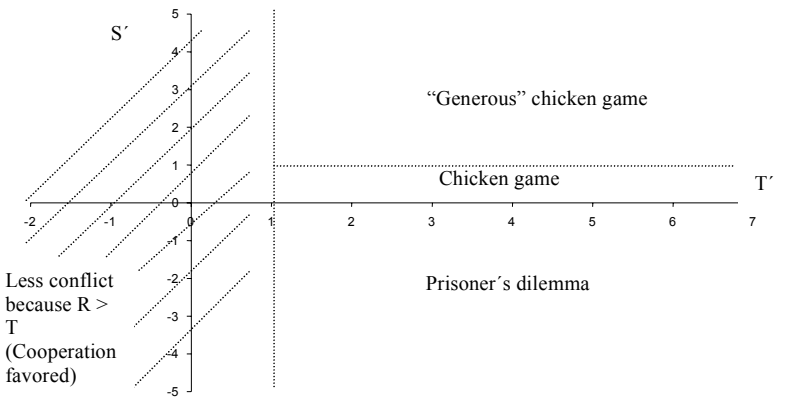

Figure 2. The areas covered by three kinds of conflicting games in a two-dimensional plane: prisoner's dilemma, chicken game and generous chicken game.

In an evolutionary context, the payoff obtained from a particular game represents the change in fitness (reproductive success) of a player. Maynard Smith [15] describes an evolutionary resource allocation within a $2 \times 2$ game as a hawk and dove game. In the matrices of Figure 1 a hawk constitutes playing D, and a dove constitutes playing C. A hawk gets all the resources playing against a dove. Two doves share the resource whereas two hawks escalate a fight about the resource. If the cost of obtaining the resource for the hawks is greater than the resource there is a CG, otherwise there is a PD. In a generous CG (not a hawk and dove game) more resources are obtained for both agents when one agent defects compared to both playing cooperate or defect.

Recent analyses have focused on the effects of mistakes in the implementation of strategies. In particular, such mistakes, usually called noise, may allow evolutionary stability of pure strategies in iterated games [8]. Two separate cases are generally considered: the trembling hand noise and misinterpretations. Within the trembling hand noise ([21], [5]) a perfect strategy would take into account that agents occasionally do not perform the intended action $^{1}$. In the misinterpretations case an agent may not have chosen the "wrong" action. Instead it is interpreted as such by at least one of its opponents, resulting in agents keeping different options about what happened in the game. This introduction of mistakes represents an important step, as real biological systems as well as computer systems will usually involve uncertainty at some level.

Here, we study the behavior of strategies in iterated games within the prisoner's dilemma and chicken game payoff structures, under different levels of noise. We first give a background to our simulations, including a round robin tournament and a characterization of the strategies that we use. We then present the outcome of an iterated population tournament, and discuss the implications of our results for game theoretical studies on the evolution of cooperation.

1. In this metaphor an agent chooses between two buttons. The trembling hand may, by mistake, cause the agent to press the wrong button.

\section{GAMES, STRATEGIES, AND SIMULATION PROCEDURES}

The PDs and CGs that we analyze are repeated games with memory, usually called iterated games. In iterated games some background information is known about what happened in the game up to now. In our simulation the strategies know the previous moves of their antagonists ${ }^{2}$. In all our simulations, interactions among players are pair-wise, i.e. a player interacts with only one player at a time

The strategies used in our iterated prisoner's dilemma (IPD) and iterated chicken game (ICG), in all 14 different strategies plus playing Random, are presented in Table 1. AllC, AllD and Random do not need any memory function at all because they always do the same thing (which for Random means always randomize). TfT and ATfT need to look back one move because they repeat or reverse the move of its opponent. Most of the other strategies also need to look back one move but may respond to defection or show forgiveness.

Axelrod ([1], [2], [3], [4]) categorized strategies as nice or mean. A nice strategy never plays defection before the other player defects, whereas a mean strategy never plays cooperation before the opponent cooperates. Thus the nice and mean terminology describes an agent's next move.

According to the categorization of Axelrod TfT is a nice strategy, but it could as well be regarded as a repeating strategy. Another category of strategies is a group of forgiving strategies consisting of Simpleton, Grofman, and Fair. They can, unlike TfT, avoid getting into mutual defection by playing cooperate. If the opponent does not respond to this forgiving behavior they start to play defect again. Finally we separate a group of revenging strategies, which retaliate a defection at some point of the game with defection for the rest of the game. Friedman and Davis belong to this group of strategies.

The set of strategies used in our simulations includes some of Axelrod's original strategies and a few, later reported, successful strategies. Of course, these strategies represent only a very limited number of all possible strategies. However, the emphasis in our work is on differences between IPD and ICG. Whether there exists a single "best of the game" strategy is outside the scope of this paper.

Mistakes in the implementation of strategies (noise) were incorporated by attaching a certain probability p between 0.02 and $20 \%$ to play the alternative action (C or D), and a corresponding probability $(1-p)$ to play the original action.

Our population tournament involves two sets of analyses. In the first set, the strategies are allowed to compete within a round robin tournament with the aim of obtaining a general evaluation of the tendency of different strategies to play cooperate and defect. In a round robin tournament, each strategy is paired once with all other strategies plus its twin. The results from the round robin tournament are used within the population tournament but will not be presented here (for the results see [10]). In the second set, the competitive abilities of strategies in iterated population tournaments were studies within the IPD and the ICG.

2. One of the strategies, Fair, also remembers its own previous moves. 
Table 1: Description of the different strategies.

\begin{tabular}{|c|c|c|}
\hline $\begin{array}{l}\text { Strat- } \\
\text { egy }\end{array}$ & $\begin{array}{l}\text { First } \\
\text { move }\end{array}$ & Description \\
\hline AllC & $\mathrm{C}$ & Cooperates all the time. \\
\hline $95 \% \mathrm{C}$ & $\mathrm{C}$ & Cooperates $95 \%$ of the time. \\
\hline $\mathrm{Tf} 2 \mathrm{~T}$ & $\mathrm{C}$ & $\begin{array}{l}\text { tit-for-two-tats, Cooperates until its opponent defects twice, } \\
\text { and then defects until its opponent starts to cooperate again. }\end{array}$ \\
\hline $\begin{array}{l}\text { Grof- } \\
\text { man }\end{array}$ & $\mathrm{C}$ & $\begin{array}{l}\text { Cooperates if } \mathrm{R} \text { or } \mathrm{P} \text { was played, otherwise it cooperates } \\
\text { with a probability of } 2 / 7 \text {. }\end{array}$ \\
\hline Fair & $\mathrm{C}$ & $\begin{array}{l}\text { A strategy with three possible states, - "satisfied" (C), } \\
\text { "apologizing" (C) and "angry" (D). It starts in the satisfied } \\
\text { state and cooperates until its opponent defects; then it } \\
\text { switches to its angry state, and defects until its opponent } \\
\text { cooperates, before returning to the satisfied state. If Fair } \\
\text { accidentally defects, the apologizing state is entered and it } \\
\text { stays cooperating until its opponent forgives the mistake } \\
\text { and starts to cooperate again. }\end{array}$ \\
\hline $\begin{array}{l}\text { Sim- } \\
\text { pleton }\end{array}$ & $\mathrm{C}$ & $\begin{array}{l}\text { Like Grofman, it cooperates whenever the previous moves } \\
\text { were the same, but it always defects when the moves dif- } \\
\text { fered (e.g.S). }\end{array}$ \\
\hline TfT & $\mathrm{C}$ & tit-for-tat. Repeats the moves of the opponent. \\
\hline Feld & $\mathrm{C}$ & $\begin{array}{l}\text { Basically a tit-for-tat, but with a linearly increasing (from } 0 \\
\text { with } 0.25 \% \text { per iteration up to iteration 200) probability of } \\
\text { playing D instead of C. }\end{array}$ \\
\hline Davis & $\mathrm{C}$ & $\begin{array}{l}\text { Cooperates on the first } 10 \text { moves, and then, if there is a } \\
\text { defection, it defects until the end of the game. }\end{array}$ \\
\hline $\begin{array}{l}\text { Fried- } \\
\text { man }\end{array}$ & $\mathrm{C}$ & $\begin{array}{l}\text { Cooperates as long as its opponent does so. Once the oppo- } \\
\text { nent defects, Friedman defects for the rest of the game. }\end{array}$ \\
\hline ATfT & $\mathrm{D}$ & $\begin{array}{l}\text { Anti-tit-for-tat. Plays the complementary move of the oppo- } \\
\text { nent. }\end{array}$ \\
\hline Joss & $\mathrm{C}$ & $\begin{array}{l}\text { A TfT-variant that cooperates with a probability of } 90 \% \text {, } \\
\text { when opponent cooperated and defects when opponent } \\
\text { defected. }\end{array}$ \\
\hline Tester & $\mathrm{D}$ & $\begin{array}{l}\text { Alters D and } \mathrm{C} \text { until its opponent defects, then it plays a C } \\
\text { and TfT. }\end{array}$ \\
\hline All D & $\mathrm{D}$ & Defects all the time. \\
\hline
\end{tabular}

A game can be modeled as a strategic or an extensive game. A strategic game is a model of a situation in which each agent chooses his plan of action once and for all, and all agents' decisions are made simultaneously while an extensive game specifies the possible orders of events. The strategic agent is not informed of the plan of action chosen by any other agent while an extensive agent can consider its plan of action whenever a decision has to be made. All the agents in our analyses are strategic. All strategies may affect the moves of the other agent, i.e. to play $\mathrm{C}$ or $\mathrm{D}$, but not the payoff value, so the latter does not influence the strategy. The kind of games that we simulate here have been called ecological simulations, as distinguished from evolutionary simulations in which new strategies may arise in the course of the game by mutation ([3]). However, ecological simulations include all components necessary for the mimicking of an evolutionary process: variation in types (strategies), selection of these types resulting from the differential payoffs obtained in the contests, and differential propagation of strategies over generations. Consequently, we find the distinction between ecological and evolutionary simulations based on the criteria of mutation rather misleading.

\section{POPULATION TOURNAMENT WITH NOISE}

We evaluated the strategies in Table 1 by allowing them to compete within a round robin tournament.

To obtain a more general treatment of IPD and ICG, we used several variants of payoff matrices within these games, based on the general matrix of Figure 3. In this matrix, C stands for cooperate; $\mathrm{D}$ for defect and $\mathrm{q}$ is a cost variable.

\begin{tabular}{|c|c|c|c|}
\hline \multirow[b]{2}{*}{ Player 1} & \multicolumn{3}{|c|}{ Player 2} \\
\hline & & C & D \\
\hline & C & 1.5 & 1 \\
\hline & D & 2 & $1.5-\mathrm{q}$ \\
\hline
\end{tabular}

Figure 3. Payoff values used in our simulation. $q$ is a cost parameter. $0<q<0.5$ defines a prisoner's dilemma game, while $q>0.5$ defines a chicken game.

The payoff for a $\mathrm{D}$ agent playing against a $\mathrm{C}$ agent is 2 , while the corresponding payoff for a $\mathrm{C}$ agent playing against a $\mathrm{D}$ agent is 1 , etc. Two $\mathrm{C}$ agents share the resource and get 1.5 each.

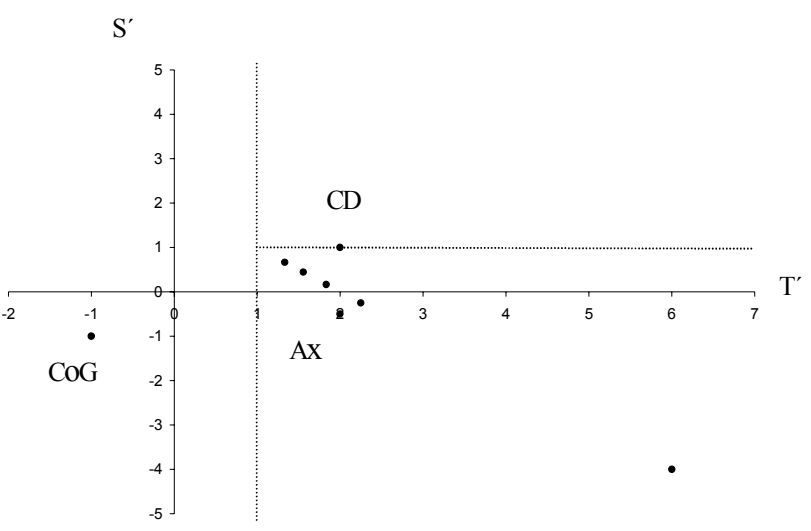

Figure 4. The different game matrices represented as dots in a 2-dimensional diagram. CoG is the coordination game, $C D$ the compromise dilemma and $A x$ is the original Axelrod game. The unmarked dots represent $0.0,0.6,0.9,1.1$ and 1.4 from upper left to lower right.

The outcome of a contest with two D agents depends on q. For $0<\mathrm{q}<0.5$, a PD game is defined, and for $\mathrm{q}>0.5$ we have a CG. Simulations were run with the values for $(1.5-q)$ set to 1.4 and 1.1 for $\mathrm{PD}$, and to $0.9,0.6$, and 0.0 for the CG (these values are chosen with the purpose to span a wide range of the games but are otherwise arbitrarily chosen). We also included Axelrod's original matrix $\mathrm{Ax}(\mathrm{R}=3, \mathrm{~S}=0, \mathrm{~T}=5$ and $\mathrm{P}=1)$ and a compromise dilemma game $\mathrm{CD}(\mathrm{R}=2, \mathrm{~S}=2, \mathrm{~T}=3$ and $\mathrm{P}=1)$. $\mathrm{A} \mathrm{CD}$ is located on the borderline between the CG area and the generous CG area. In the discussion part we also compare the mentioned strategies with a coordination game $\operatorname{CoG}(\mathrm{R}=2, \mathrm{~S}=0, \mathrm{~T}=0$ and $\mathrm{P}=1)$, the only game with $T^{\prime}<1$. CoG is included as a reference game and does not belong to the conflicting games. In Figure 4 all these games are shown within the two-dimensional plane. The $\mathrm{CD}$ is closely related 
to the chicken game and $\mathrm{CoG}$ is a game with two Nash equilibria, playing $(C, C)$ or playing $(D, D)$. In Johansson et al. [11] a further discussion about $\mathrm{CD}$ and $\mathrm{CoG}$ is found. Each game in the tournament was played on average 100 times (randomly stopped) ${ }^{3}$ and repeated 5000 times.

In the second part of the simulation, strategies were allowed to compete within a population tournament for the iterated games. These simulations were based on the same payoff matrices for IPD and ICG as in the initial round robin tournament. Based on the success in the single round-robin tournaments, strategies were allowed to reproduce copies into the next round robin tournament, creating a population tournament, i.e. a quality competition in the roundrobin tournament (make a good score) is transformed to an increased number of copies in the population tournament. Each of the fifteen strategies starts with 100 copies resulting in a total population of 1500 . The number of copies for each strategy changes, but the total of 1500 copies remains constant. The proportions of the different strategies propagated into a new generation were based on the payoff scores obtained in the preceding round-robin tournament. A given strategy interacts with the other strategies in the proportions that they occur in their global population. The games were allowed to continue until a single winning strategy was identified, i.e. the whole population consists of the same strategy, or until the number of generations reached 10,000. In most of the simulations, a winning strategy was found before reaching this limit.

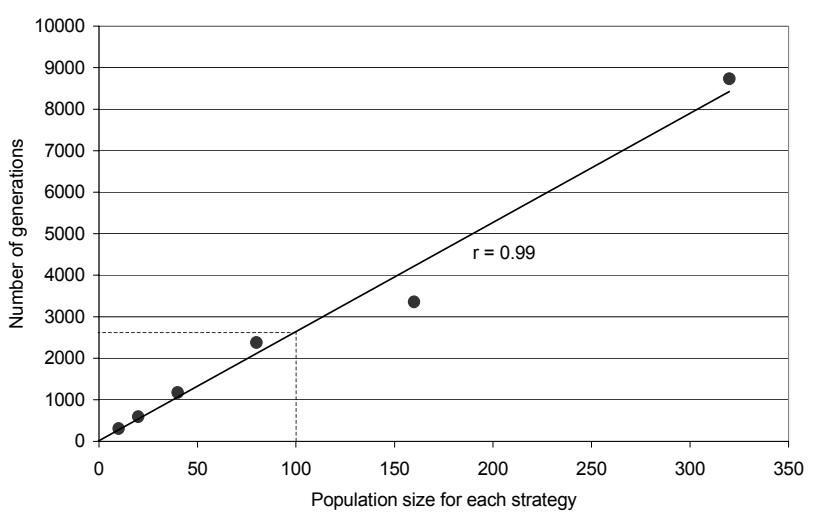

\section{Figure 5. Number of generations for finding a winning strategy among 15 random strategies with a varying population size.}

Also, if a pure population of agents with the random strategy are allowed to compete with each other in a population game, a single winning strategy will be found after a number of generations. This has to do with genetic drift and small simulation variations between different agents in their actual play of $\mathrm{C}$ and $\mathrm{D}$ moves. As seen in Figure 5, with increased total population size of agents the

3. If an agent knows exactly or with a certain probability when a game will end, it may use such information to improve its behavior. Because of this, the length of the games was determined probabilistic, with an equal chance of ending the game with each given move (see also [1]) number of generations for finding a winning strategy increases. This almost linear increase $(r=0.99)$ is only marginally dependent of what game is played.

The simulation consists of 15 random strategies with a population size of 100 individuals each, i.e. small differences between strategies will favor/be unfair to a certain strategy. Randomized strategies with 100 individuals are, according to Figure 5., supposed to halt, i.e. all 1500 individuals belonging to the same initial strategy, after approximately 2800 generations in a population game. Which strategy that wins will vary between the games. There are two possible kinds of winning strategies: pure strategies that halt, and mixed strategies (two or more pure strategies) that do not halt. If there is an active choice of a pure strategy it should halt before 2800 generations, because otherwise playing random could be treated as a winning pure strategy. There is no reason to believe that a single strategy winner should be found by extending the simulation beyond 10000 generations. If there exists a pure solution, this solution should turn up much earlier.

The effect of uncertainty (noise) in the choice of actions (C or D) by the agents within the tournaments was analyzed by repeating the tournaments in environments of varying levels of noise. Tournaments were run at $0,0.02,0.2,2$, and $20 \%$ noise. The probability of making a mistake was neither dependent on the sequence of behaviors up to a certain generation, nor on the identity of the player. Noise will affect the implementation of all strategies except for the strategy Random. We focused on three different aspects when comparing the IPDs and ICGs, which will be further analyzed in the discussion part:

1. The number of generations for finding a winning strategy.

2. Differences in robustness for the investigated strategies.

3. The behavior of the generally regarded cooperative strategy TfT in IPD and ICG.

\section{RESULTS}

In Figure 6 and Figure 7 the success of individual strategies in IPD, ICG and CD population games at no noise and $0.2 \%$ of noise are shown. The repeating strategy TfT is represented by a solid line, the forgiving strategies Simpleton, Grofman, and Fair by dashed lines, and the revenging strategies Friedman and Davis by dotted lines.

In the IPD games TfT, Friedman and Davis are the most successful with no noise (Figure 6), while TfT, Grofman, Fair and Friedman are the most successful with $0.2 \%$ noise (Figure 7). For the other levels of noise (not shown in figures) TfT, and for Axelrod's matrix also Tf2T, is dominating with $0.02 \%$. With $2 \%$ noise Davis and TfT dominates, and finally AllD and Friedman are the dominating strategies with $20 \%$ noise.

At no noise all three groups of strategies are approximately equally successful in ICG (Figure 6), with a minor advantage for the forgiving strategies Simpleton, Grofman, and Fair. This advantage increases with increasing noise. The revenging strategies Friedman and Davis disappear at $0.02 \%$ noise and TfT at $0.2 \%$ noise (Figure 7) leaving the forgiving strategies alone at $0.2 \%$ and $2 \%$ noise. At $20 \%$ noise AllD supplements the set of successful strategies. 


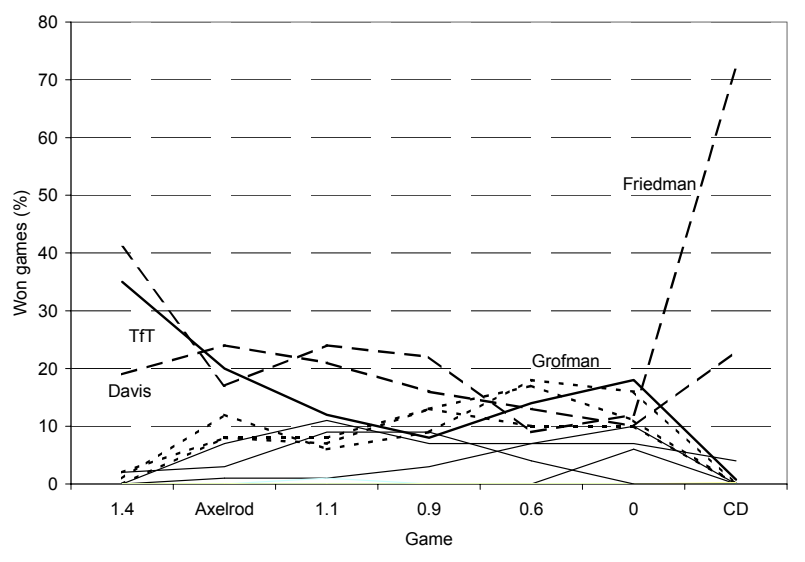

Figure 6. Percentage of runs won by strategies in the population games for different chicken games $(0.9,0.6,0)$, prisoner's dilemmas $(1.4, A x, 1.1)$ and the compromise dilemma with $0 \%$ noise.

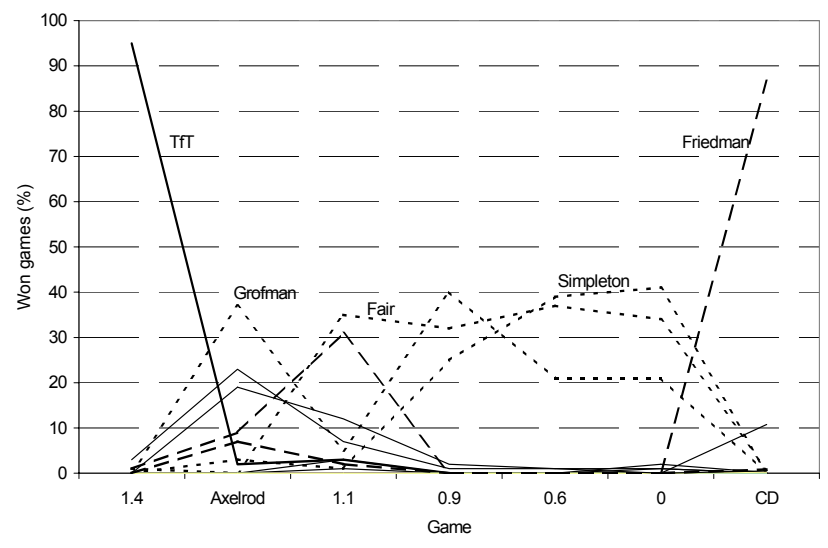

Figure 7. Percentage of runs won by strategies in the population games for different chicken games $(0.9,0.6,0)$, prisoner's dilemmas $(1.4, \mathrm{Ax}, 1.1)$ and the compromise dilemma with $0.2 \%$ noise

The revenging strategies Friedman and Davis completely outperform Simpleton, Grofman, Fair and TfT strategies in CD. With increasing noise ATfT (0.2-20\% noise) and AllD (20\% noise) become more successful as part of a mixed set of strategies, because $\mathrm{CD}$ does not find a single winner (Figure 8).

Finally, in CoG Tf2T and TfT are dominating with $0 \%$ noise. Tf $2 \mathrm{~T}$ together with AllC and Grofman constitute all the winning strategies with $0.02 \%, 0.2 \%$ and $2 \%$ noise. $95 \% \mathrm{C}$ is the only winner with $20 \%$ noise.

With increased noise the group of Simpleton, Grofman, and Fair become more and more successful in ICG up to and including $2 \%$ noise. When noise is introduced, IPDs favor the repeated TfT. With increased noise the revenging Friedman and Davis disappears for both ICG and IPD. Finally, with $20 \%$ noise AllD is the dominating strategy. More and more defecting strategies will dominate with increasing noise in IPD. Finally in CD the revenging strategies Friedman and Davis dominates. In contrast to IPD and CD cooperating and forgiving strategies dominate in ICG which makes the ICG the best candidate for finding robust strategies.

On average there was $80 \%$ accordance (for all levels of noise) between winning strategies in different ICG, i.e. four out of five strategies being the same. In the IPD there was a discrepancy with only on average $35 \%$ of the winning strategies being the same. The performance of the 0.4 and Ax matrices are similar within the ICG. This was especially notable for both matrices without noise (on average $75 \%$ ) and for the 0.4 matrices with 2 and $20 \%$ noise (on average $55 \%$ ).

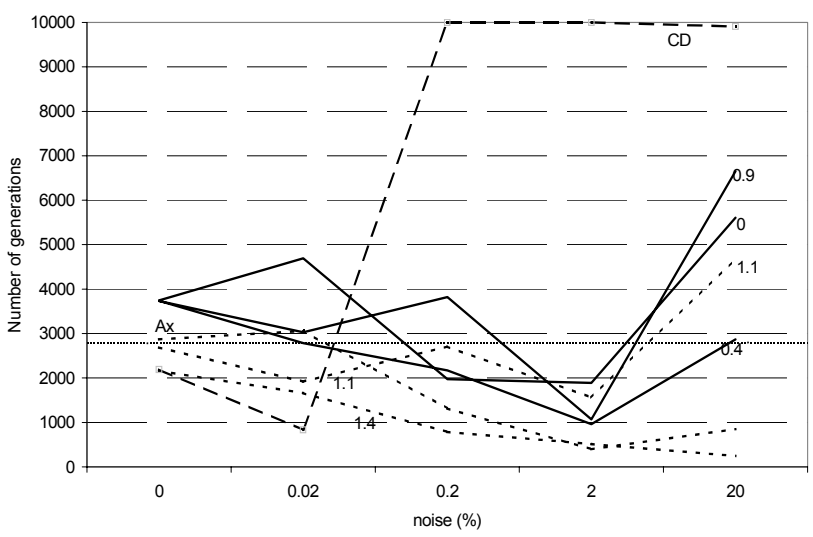

Figure 8. Number of generations for finding a winning strategy in chicken games, prisoner's dilemmas and compromise dilemma at different levels of noise.

In Figure 8, the number of generations needed to find a winning strategy is plotted for different level of noise. The dotted line shows the expected generations (2800) for competing Random strategies mentioned earlier. At 0 or low levels of noise more generations are needed in the ICG for finding a winner than in IPD. The lowest numbers of generations are needed with $2 \%$ of noise and the highest with $0 \%$ and $20 \%$ noise. There is no single strategy winner for the $\mathrm{CD}$ game with $0.2 \%$ noise and above.

In summary; coordination games give mutual cooperation the highest results, which favors nice, but to a less extent forgiving, strategies. Compared to the ICG, IPD is less punishing towards mutual defection, which allows repeating and revenging strategies to become more successful. Finally in the compromise dilemma, where playing the opposite to the opponent is favored, revenging and/or a mixture of different strategies are favored. With increased noise $(2 \%$ or below), forgiving strategies become more and more successful in ICG while repeating and revenging strategies are more successful in IPD.

\section{DISCUSSION}

In our investigation we found ICG to be a strong candidate for being the major cooperate game. ICG seems to facilitate cooperation as much as or even more than IPD, especially under noisy conditions. Axelrod ([1], [2], [3]) regarded TfT to be a leading 
cooperative strategy, but in our investigation we found TfT to have poor success under noisy conditions within ICG. These statements will be further addressed in the discussion below.

Most studies today consider the IPD as a cooperative game where nice and forgiving strategies are successful. A typical winning strategy, like TfT, ends up as an agent playing cooperate all the time. There are contradictory arguments about cooperation within chicken games. The advantage of cooperation may be expected to be stronger, because the cost of defection is higher than in the prisoner's dilemma. Lipman [13] suggests that in ICG, mutual cooperation is less clearly the best outcome because there is no dominant strategy. Each agent prefers the equilibrium in which she defects and the other cooperates, but has no way to force the other agent to cooperate. A mixed strategy or a set of strategies, unlike a single dominant strategy, may favor mutual cooperation. With pure and mixed strategies we here refer to the set of strategies (played by individuals) winning the population tournament. A mixed strategy is a combination of two or more strategies from the given set of strategies i.e. an extended strategy set could include the former mixed strategy as a pure strategy.

We think an advantage of cooperation for the chicken games can be explained by a greater robustness. Within a machine vision context, a system is robust if it does not malfunction in the presence of disturbances. This robustness may be present if more strategies are allowed and/or noise is introduced. For all investigated ICG without any noise present, more generations were needed for finding a winner compared to IPD. With minor exceptions this is also true for noise between $0.02 \%$ and $20 \%$. In Carlsson [10] the same conclusion was reached for a large set (more than 200) of games with variants of AllD, AllC, TfT and ATfT.

If it is true that more cooperating strategies are favored in ICG, we should also expect nice and forgiving strategies to be successful in this game. In the ICG, both players that play defect fare the worst, which should favor forgiving strategies. Both ICG and coordination game favors nice, non-revenging, strategies, but unlike coordination game ICG may forgive a defection from the opponent. This makes ICG a primary candidate for being the main cooperative game, favoring both niceness and forgivingness.

An interesting exception to the higher success of cooperating strategies within ICG is the poor success under noisy conditions of TfT. The vulnerability of TfT to errors in the implementation of actions within the IPD is well known and has been discussed extensively ([3], [16], [5], [23], [7], [18], [19]). The even poorer ability of TfT to handle noise within the ICG, is however a novel finding. The classical description by Axelrod [3] of a successful strategy in a deterministic (non-noisy) environment is that it should be nice (not be the first to defect), provocable (immediately punish defection), forgiving (immediately reciprocate cooperation), and simple (easily recognizable). Obviously, under noisy conditions TfT either behaves less nice, provocable, forgiving, and simple, or these characteristics are of less value in the ICG. Axelrod and Dion [5] suggested that the difficulty for TfT to handle noise is an inherent consequence of generosity: vulnerability to exploitation. Errors in the implementation of strategies give rise to unconditional cooperation, which undercuts the effectiveness of simple and reciprocating strategies. It also introduces mutual defection among TfT players, reducing their obtained payoffs [19]. In the long run, the average payoffs of two interacting TfT players in a noisy environment converge to that of two interacting Random players [16].
Thus, the main problem for TfT in a noisy environment may be to cope with copies of itself.

A solution to the problem of noise for a strategy is to punish defection in the other player less readily than does TfT. This can be done either by not immediately responding to an opponent's defection or by avoidance of responding to the other player's defection after one has made an unintended defection ([16]; see also [23]). Thus, some modified versions of TfT, Contrite tit-for-tat (CTfT) and generous tit-for-tat (GTfT) have proved to cope much better with noise than the original TfT ([23], [8)). Bendor [6] concludes that uncertainty sometimes affects nice strategies negatively but he also proposes that reciprocating but untrustworthy strategies may start to cooperate because of unintended actions.

Several attempts have been made to classify strategies according to their willingness to play cooperate and defect, respectively, the classical being Axelrod's [1] distinction between nice and mean strategies based on whether a strategy's first draw is cooperate or defect, respectively. Under noisy conditions, the static description of a strategy based on its behavior under non-noisy becomes more or less meaningless. Naturally, a nice strategy then becomes meaner, and a mean strategy becomes nicer, but the actual behavior is difficult to evaluate.

In our opinion, the discussion about the evolution of cooperative behavior has relied too heavily on analyses within the prisoner's dilemma context. The differences in the outcome of IPD and ICG shown in our study suggest that future game theoretical analyses on cooperation should explore alternative payoff environments. The chicken game was discussed as a special case within the general hawk and dove context by Maynard Smith [15], but for some reason subsequent game theoretical studies has almost exclusively focused on the prisoner's dilemma. This is unfortunate, since the chicken game appears to us to be a very interesting game in explaining the evolution of cooperative behavior. If we give the involved agents the ability to establish trust the difference between the two kinds of games are easier to understand. In the PD establishing credibility between the agents means establishing trust, whereas in CG, it involves creating fear, i.e. avoiding situations where there is too much to lose[22]. This makes ICG a strong candidate for being a major cooperate game together with IPD. We therefore hope that in future studies, more attention will be paid to the role of chicken games in the evolution of agents with cooperative behavior within multi agent systems.

\section{ACKNOWLEDGEMENTS}

We are grateful to Magnus Boman, Paul Davidsson, Roger Härdling, Stefan Johansson, Kristian Lindgren, Michael Mattsson, Per Lundberg, David Sloan Wilson, and anonymous referees for valuable comments on (different versions of) the manuscript and Martin Hylerstedt for proofredding. K. I. J. was supported by the Swedish Natural Science Research Council.

\section{REFERENCES}

[1] Axelrod, R. 1980a. Effective Choice in the Prisoner's Dilemma. J. Confl. Resol., 24: 3-25

[2] Axelrod, R. 1980b. More Effective Choice in the Prisoner's Dilemma. J. Confl. Resol., 24: 379-403.

[3] Axelrod, R. 1984. The Evolution of Cooperation. New York: Basic Books. 
[4] Axelrod, R. and Hamilton, W.D. 1981. The evolution of cooperation. Science, 211: 1390-1396.

[5] Axelrod, R. and Dion, D. 1988. The further evolution of cooperation. Science, 242: 1385-1390.

[6] Bendor, J. 1993. Uncertainty and the evolution of cooperation. J. Conflict Resolut., 37: 709-734.

[7] Bendor, J., Kramer, R.M. and Stout, S. 1991. When in doubt: Cooperation in a noisy Prisoner's Dilemma. J. Conflict Resolut., 35: 691-719.

[8] Boyd, R. 1989. Mistakes allow evolutionary stability in the repeated Prisoner's Dilemma game. J. theor. Biol., 136: 4756.

[9] Carlsson B. and Johansson, S. 1998. An Iterated Hawk-andDove Game. In Agents and Multi-agent Systems. Lecture Notes in Artificial Intelligence 1441 (W. Wobcke, M. Pagnucco, and C. Zhang, eds), pp. 179-192, Berlin: SpringerVerlag.

[10] Carlsson, B., 2001, Simulating how to Cooperate in Iterated Chicken Game and Iterated Prisoner's Dilemma, in eds. Liu, J., Zhong, N., Tang, Y.Y., and Wang, P.S.P., Agent Engineering, Series in Machine Perception and Artificial Intelligencevol 43, World Scientific, Singapore.

[11] Johansson, S., Carlsson, B., and Boman, M. 1998. Modeling strategies as Generous and Greedy in Prisoner's Dilemmalike games. 1998 Proceedings from the Second Asia Pacific Conference on Simulated Evolution and Learning (SEAL98), Canberra.

[12] Koeslag, J.H. 1997. Sex, The Prisoner's Dilemma Game, and the Evolutionary Inevitability of Cooperation. J. theor. Biol., 189: 53-61.

[13] Lipman, B.L. 1986. Cooperation among egoists in Prisoner's Dilemma and Chicken Game. Public Choice, 51: 315-331.

[14] Luce, R.D., and Raiffa, H. Games and Decisions Dover Publications Inc. 1957.

[15] Maynard Smith, J. 1982. Evolution and the theory of games. Cambridge: Cambridge University Press.

[16] Molander, P. 1985. The optimal level of generosity in a selfish, uncertain environment. J. Conflict Resolut., 29: 611-618.
[17] Nishimura, K. and Stephens D.W. 1997. Iterated Prisoner's Dilemma: Pay-off Variance. J. theor. Biol., 188: 1-10.

[18] Nowak, M.A. and Sigmund, K. 1992. Tit for tat in heterogeneous populations. Nature, 355: 250-253.

[19] Nowak, M.A. and Sigmund, K. 1993. A strategy of win-stay, lose-shift that outperforms tit-for-tat in the Prisoner's Dilemma game. Nature, 364: 56-58.

[20] Rapoport, A. and Chammah, A.M. 1965. Prisoner's Dilemma: A study in Conflict and Cooperation. Ann Arbor: University of Michigan Press.

[21] Selten, R., Reexamination of the perfectness concept for equilibrium points in extensive games. International Journal of Game theory, 4:25-55, 1975.

[22] Snyder, G. 1971. "Prisoner's dilemma" and "Chicken" models in international politics. Int. Stud. Quart.,15: 66-103.

[23] Wu, J. and Axelrod, R. 1995. How to cope with noise in the iterated Prisoner's Dilemma. J. Conflict Resolut., 39: 183189.

Bengt Carlsson (1951-) defends his Ph. D. thesis (Conflicts in Information Ecosystems, Blekinge Institute of Technology 2001, ISBN 91-7295-005-6) in December 2001 at Blekinge Institute of Technology. His research focuses on conflicting games and different models of information ecosystem using a conflicting approach.

K. Ingemar Jönsson (1959-) is docent and assistant professor in theoretical ecology at Lund University. His research focuses on general problems within the field of evolutionary ecology, in particular the evolution of life histories (patterns of age-specific reproduction and survival). His $\mathrm{PhD}$-thesis (Costs and tactics in the evolution of reproductive effort, Lund University 1996, ISBN 917105-073-6) included both theoretical and empirical work on optimal reproductive investment and costs of reproduction. More recently he has worked on microevolutionary aspects of the evolution of ametabolic life stages (cryptobiosis). In his research, K. Ingemar Jönsson attempts to combine theoretical and conceptual analyses with experimental work both in laboratory and under field conditions. 